\title{
HEART MEASUREMENTS OF NON-HUMAN PRIMATES BY RADIOGRAPHIC TECHNIQUES
}

\section{MENSURAÇÃO CARDÍACA DE PRIMATAS NÃO HUMANOS POR MEIO DE TÉCNICAS RADIOGRÁFICAS}

\author{
Mari Jane Taube ${ }^{1^{*}}$ ORCID - http://orcid.org/0000-0001-7755-2960 \\ Luciana do Amaral Oliveira' ${ }^{1}$ ORCID - http://orcid.org/0000-0002-5149-4229 \\ Patricia Santos Rossi ${ }^{1}$ ORCID - http://orcid.org/0000-0002-5869-6624 \\ Andressa Hiromi Sagae ${ }^{1}$ ORCID - http://orcid.org/0000-0001-9144-4624 \\ Ricardo Coelho Lehmkuhl ${ }^{1}$ ORCID - http://orcid.org/0000-0002-0932-0305 \\ Zara Bortolini' ${ }^{1}$ ORCID - http://orcid.org/0000-0002-9417-5949 \\ ${ }^{1}$ Universidade Estadual do Centro Oeste- UNICENTRO, Guarapuava, PR, Brasil. \\ "Correspondent author - taubemarijane@gmail.com
}

\begin{abstract}
Thoracic Radiology is a diagnostic technique used to study several aberrations, including cardiac abnormalities, which can be determined by VHS (vertebral heart size). The heart length and width are measured, and the CTR (cardiothoracic ratio) is calculated, by comparing the heart width to the thoracic diameter. This study aimed at measuring the cardiac silhouette of non-human primates (PNH) belonging to the genus Sapajus sp., and the Alouatta guariba species through VHS and CTR. From among the eight animals assessed, it was evident that in both the Sapajus sp. and the Alouatta guariba, the highest HSV change index was observed, depending upon the age of the animal. However, although the individuals of the Alouatta guariba species are bigger in size and heavier, the animals of the genus Sapajus sp. have a larger heart size in proportion to their body.
\end{abstract}

Key words: Heart, thoracic diameter, radiology, heart silhouette.

\section{Resumo}

O uso da radiologia torácica surge como forma de diagnóstico para inúmeras anormalidades, inclusive cardíacas, as quais podem ser avaliadas por métodos VHS (vertebral heart size), realizado a partir da mensuração do comprimento e largura do coração e CTR (cardiothoracic ratio), o qual compara a largura do coração, pelo diâmetro torácico. Assim, objetivamos mensurar a silhueta cardíaca de primatas não humanos (PNH) do gênero Sapajus sp. e espécie Alouatta guariba por meio do VHS e CTR. Dos oito animais avaliados, percebeu-se que tanto nos Sapajus sp. e nos Alouatta guariba, o índice de maior alteração do VHS foi encontrado pela idade do animal. E que apesar dos animais da espécie Alouatta guariba serem maiores e mais pesados, proporcionalmente ao corpo, os animais do gênero Sapajus sp. possuem um maior tamanho cardíaco.

Palavras-chave: Coração; diâmetro torácico; radiologia; silhueta cardíaca. 


\section{Introduction}

Under radiographic examination, the thorax is visible in diagnostic form, which facilitates the determination and diagnosis of cardiac diseases or any type of changes ${ }^{(1)}$. The radiographic examination must necessarily always follow a thorough clinical evaluation, which must include historical clinical, physical and laboratorial examinations to achieve a complete diagnosis ${ }^{(2)}$.

Veterinary Medicine already widely utilizes this form of examination because it is a non-invasive procedure. It enables the structures to be visualized and therefore the thoracic abnormalities can be identified $^{(3)}$.

The chest x-ray offers critical data on the dimensions of the cardiac chambers, pulmonary parenchyma, upper airway abnormalities, and chest wall abnormalities, besides others ${ }^{(4)}$.

In Veterinary Medicine, it is crucial to measure the heart size to evaluate the patients with heart disease; heart failure was observed to occur more commonly when the animals reached middle age, although the risk of a particular type of heart disease developing will vary according to the breed ${ }^{(5)}$. However, to assess the cardiac silhouette, the normal heart patterns relative to its size and position are mandatory, taking into account both chest conformation and patient age. Dogs reveal a variety of thoracic conformations, a factor that confuses the comparative analysis of the cardiac silhouette. It is also noteworthy that the body experiences changes relative to its size and position over time ${ }^{(1)}$.

Veterinary Medicine has been increasingly investigating wild animals, and radiographic examination seems to be a good method of assessing several organs, including the heart. It facilitates the acquisition of normality patterns to characterize the abnormalities as well as to clarify several pathological processes inherent to the species ${ }^{(6)}$.

To enable the assessment of the cardiac silhouette, the VHS method (vertebral heart size) was created. This is a quantitative measurement that predicts the condition of the heart through the performance of latero-lateral radiographs utilizing the vertebral unit system. Thus, the lengths of the thoracic vertebrae are compared with the measurements drawn from the cardiac silhouette. In some domestic animals, like dogs, for instance, these values have already been established $\operatorname{prior}^{(1)}$. However, in general, for wild animals including HNP, such data are scarce in the literature.

The CTR measurement, which influences the cardiothoracic ratio, is taken by comparing the widest widths of the cardiac silhouette with the rib cage diameter at the height of the eighth rib pair; also, the ventro-dorsal incidences with the animal aligned are necessary ${ }^{(7)}$.

Due to the paucity of literature in terms of cardiac measurements by specific radiographic techniques for non-human primates, and related to the fact that the veterinarian's practice is increasingly growing in the medical and surgical clinic of wild animals, this study intends to study the cardiac measurements of the genus Sapajus sp. and Alouatta guariba species in order to deform a database.

\section{Material and methods}

With the approval from CEUA-Unicentro, this project was supported by the Official Letter number $003 / 2014$ and because of the need for anesthetic protocol for these animals to collect the CSF for extra analyses, they passed, at the same time, the realization for radiographic examinations.

Eight clinically healthy, Non-Human Primates (HNP), having a nearly two-year history of management 
in captivity, were assigned to the Wild Animal Care Sector (SAAS) of Unicentro - Paraná, three of the genus Sapajus sp. (one male and two females) and five individuals of the Alouatta guariba species (a male and female males), between the young and adult stages.

The x-rayed animals were first physically contained by capture within the enclosure they occupied and then subjected to the chemical containment procedure. The anesthetic protocol employed involved utilizing Ketamine Hydrochloride $(10 \mathrm{mg} / \mathrm{kg}$ ) and Xylazine Hydrochloride $(0.5 \mathrm{mg} / \mathrm{kg})$. Monitoring of the cardiac and respiratory functions, as well as body temperature was performed throughout the duration of the anesthetic.

The radiographic examinations were performed keeping the animals in the ventral decubitus (RV) and right lateral (LLD) positions to enable both HSV and CTR measurements to be taken. These animals were imaged with radiology equipment, Brand CDK, Model Mascote 100/200 and radiographic film brand FUJI sizes $24 \times 30$ or 30x40. This device is available in the Diagnostic Imaging Service of the Clinical Veterinary School (CEVET) in the University State of the Western Center - UNICENTRO / PR. The apparatus was calibrated using the $45 / 50 \mathrm{kV}, 200 \mathrm{~mA}$ and the 0.05 second technique.

VHS was measured using the protocol that determines the cranio-caudal dimensions - organ width (L) established from the measurement at the position of the ventral part of the caudal vena cava, in the caudal margin of the heart, up to its cranial border. Next, the dorso-ventral dimension - length (C) was measured, or the long axis was drawn from the measurement taken from the carina or bronchial bifurcation, up to the left ventricular apex. It must be noted that the meeting point of the two axes needed to form a $90^{\circ}$ angle.

Once these two measurements were taken, the position of the cranial border of the fourth thoracic vertebra was identified and using this the number of vertebrae corresponding to the measure of (C) was recorded; the same process was repeated to measure the (L). After obtaining the estimated number of vertebrae of $(\mathrm{C})$ and $(\mathrm{L})$ the sum of these two values, the final measurement in the HSV unit was done. This implies VHS $=\mathrm{C}+\mathrm{L}$, measured as recorded in Figure 1, based on the protocol of Buchanan and Bücheler ${ }^{(1)}$.

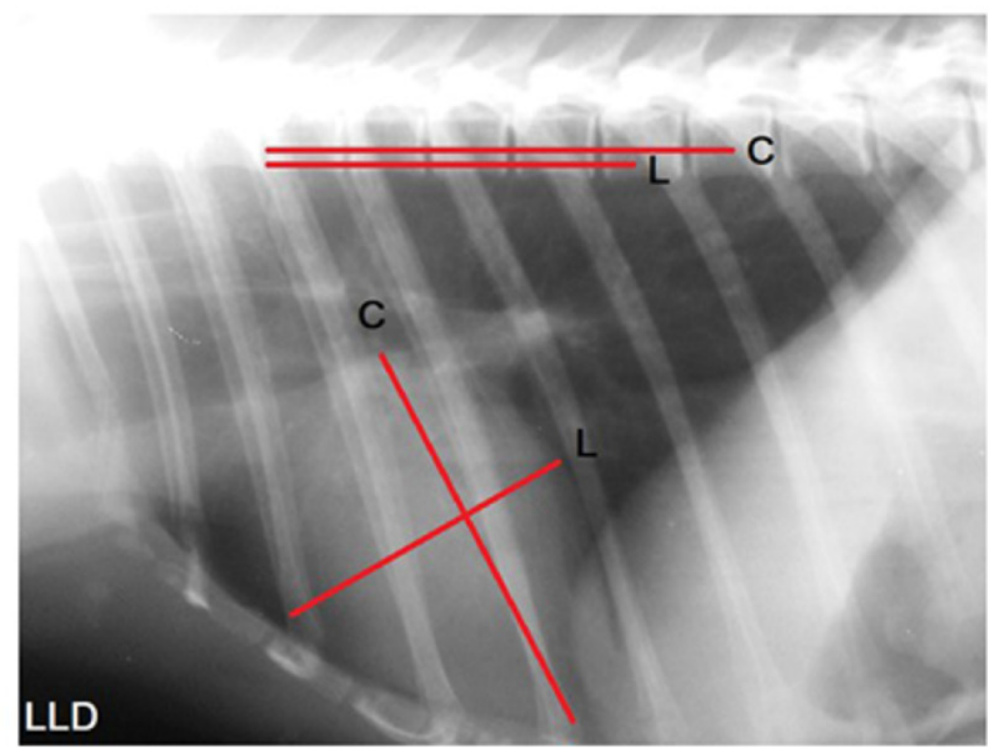

Figure 1. The right lateral chest $\mathrm{x}$-ray of Alouatta guariba with the measurements of the long axis (C) and short axis (L) of the heart, transferred to the spine commencing at the cranial border of the fourth thoracic vertebra. 
Using the methodology of Schillaci(7) heart size (CTR) was assessed by comparing the heart widths; these measurements were taken from the vertical line (spinal processes of the thoracic vertebrae) to the right side (MR) and left side (ML) at its largest diameter point, while the thoracic diameter was measured at the height of the eighth rib pair. The CTR measurement was based on the sum of the MR and ML by thoracic diameter (MTD)(7), that is, CTR = MR + ML / MTD, as shown in Figure 2. It is significant that this measurement was done solely for the animals of the genus Sapajus sp.

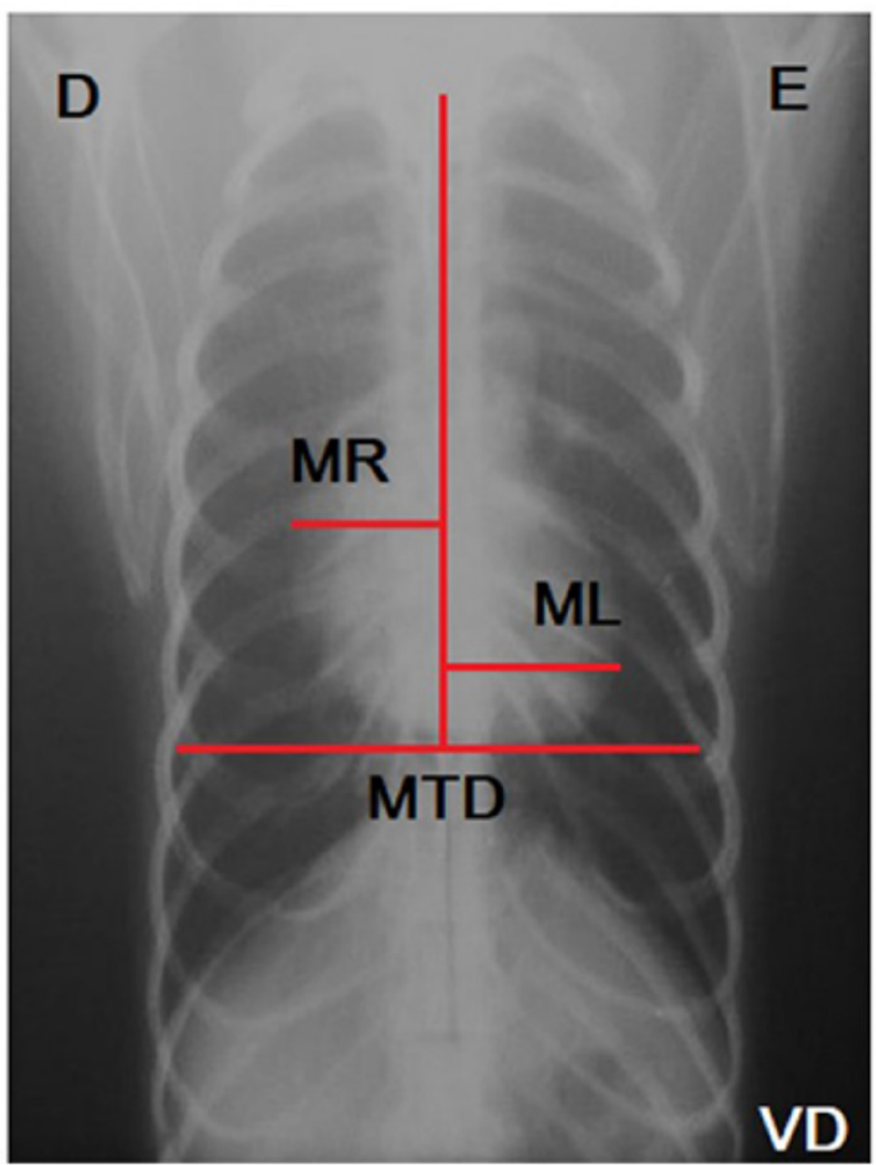

Figure 2. Ventro-dorsal thoracic-ventro view of Sapajus sp. and the measurements of $\mathrm{MR}+\mathrm{ML}$ and MTD obtained, to measure the CTR.

\section{Results and discussion}

The animals investigated in this study revealed a cardiac silhouette between the third and fifth intercostal space, on lateral projection. The animals of the Alouatta guariba species, in particular, have their left heart axis, rendering it hard to record the CTR measurement.

In the individuals of the Sapajus sp. the heart was found to be in the mediastinum center. The males registered a HSV value of 10.7, while the females showed a mean value of 9.8 (Table 1). For the CTR measurements, the values recorded were 0.58 and 0.61 for the females and 0.65 for young males.

In the Alouatta guariba species, the animals displayed the heart positioned between the third and fifth intercostal space; the HSV of the males showed a mean of 9.4, while that of the females was 9.3 (Table 1). 
Table 1. Identification of the individual animals of the Sapajus sp., and the measurements obtained via the HSV and CTR methods, as well as the Alouatta guariba individuals with the HSV values determined

\begin{tabular}{cccccc}
\hline Animal & Sex & Age & Weight kg) & VHS & CTR=MR +ML /MTD \\
\hline Sapajus sp. & F & Adult & 2.380 & 9.8 & $1.6+1.85 / 5.9=\mathbf{0 . 5 8}$ \\
Sapajus sp. & M & Young & 2.065 & 10.7 & $1.7+2.2 / 6.0=\mathbf{0 . 6 5}$ \\
Sapajus sp. & F & Adult & 2.100 & 9.9 & $1.65+1.9 / 5.8=\mathbf{0 . 6 1}$ \\
Alouatta guariba & F & Young & 3.800 & 9.3 & - \\
Alouatta guariba & M & Adult & 5.300 & 8.7 & - \\
Alouatta guariba & M & Adult & 5.400 & 9.6 & - \\
Alouatta guariba & M & Adult & 5.500 & 10.0 & - \\
Alouatta guariba & $\mathrm{M}$ & Adult & 5.600 & 10.6 & - \\
\hline
\end{tabular}

On completion of the morphological assessment of the Sapajus sp. it is clear that the adult females did not show great variations in the outcomes, similar to those reported in another study done with the Sapajus sp. ${ }^{(8)}$ or Callithrix jacchus ${ }^{(9)}$ and even in Macaca mulatta ${ }^{(10)}$; this enabled the conclusion to be drawn that healthy adult female primates with close body shape, do not show large variations among the measurements recorded.

In Callithrix jacchus no differences were registered between females and males ${ }^{(9)}$. However, in the study in question, the difference between the genders was pivotal. It is noteworthy that the young males recorded higher HSV values than did the adult females, thus suggesting that prior to determining the sex of the animal, it is mandatory to know the patient's age. This will enable ascertaining what is normal for the abnormal; this is chiefly because of the likelihood of normal organomegaly occurring in the young individuals. A study done using Macaca fascicularis, showed differences in heart width, linked to age and sex, and only with age in relation to length ${ }^{(11)}$. The animals of genus Sapajus sp. exhibited measurements close to the VHS values of dogs (up to 10.5).

In their study, Alves et al. ${ }^{(12)}$, reported HSV values in Cebus apella to be around 9.7, while RochaNeto et al. ${ }^{(8)}$, also recorded CTR values $(0.55+/-0.04)$; the animals used also showed similarities in the variations of sex and weight ${ }^{(12)}$. The values established for the species Macaca tonkeana (0.59) ${ }^{(7)}$, Macaca nigra $(0.58)^{(7)}$ and Macaca fascicularis $(0.55 \text { to } 0.58)^{(7,11)}$ validate the CTR measures identified in the present study, thus enabling the findings of cardiothoracic measurements among the species to be correlated. It is noteworthy that the analysis of the young male in question exhibits a higher HSV value; a difference in the CTR is also seen when females are compared, a factor that highlights the significance of analyzing the age of the animals assessed.

Published data dealing with CTR measurements in non-human primates are rather scarce. The species Cercopithecus neglectus has been reported to have a CTR value of 0.68 ; however, these have recorded the incidence of heart disease ${ }^{(13)}$. When the CTR data found in HNP are compared with that of humans, it is evident that values higher than 0.55 are already accepted to be indicative of cardiomegaly ${ }^{(14)}$. 
In connection with the Alouatta guariba species, the heaviest males revealed the highest VHS, while the young females also showed higher HSV in relation to the male with lower weight. From a cardiac study conducted using the same species an average HSV of 9.04 was recorded, while the other data still with reference to the Callimico goeldii species registered an average HSV of 9.35; the current study too reveals an average of 9.35 for VHS units(15).

Contrary to the Sapajus sp., in the Alouatta guariba the anatomical presentation of the heart in the thoracic cavity occupies only the left hemithorax, similar to the Lemur catta ${ }^{(16)}$. It is different from that of the Chlorocebus sabaeus species in which the cardiac base is situated in the right hemithorax ${ }^{(17)}$, a factor that precludes the relation of the CTR derived post ventrodorsal radiography, as performed for the genus Sapajus sp.

Thus, it is evident that age is the most pertinent factor, and young animals may exhibit a higher HSV and CTR, arising from the proportion of their rib cage, with the heart still in adjustment, given that it is obvious in both groups.

When the animals were compared, the weight was analyzed and the animals belonging to group Sapajus sp. were seen to have lower weight and, therefore were smaller; however, the HSV value was higher.

\section{Conclusion}

From the findings of this study, it was inferred that the age of the animals within their own species was the pivotal factor when considering the cardiac silhouette; here the young animals exhibited a higher HSV, a factor that must be given importance because of the total non-growth of their rib cage.

Another conclusion drawn was that the animals of the Sapajus sp. expressed a proportionally larger size of the cardiac silhouette than did those of the Alouatta guariba species, in which they heavier and larger sized and the HSV is proportionally lower.

For the genus Sapajus sp. the measurements from the CTR are also affected by the age of the animal; incidentally, young animals have higher CTR values.

The addition of the results in this study linked to the cardiac size and the clinical state of the animals indicates that the animals from both species were healthy, and had no obvious cardiac alterations, thus establishing reference values for both specimens.

\section{References}

1. Buchanan JW, Bücheler J. Vertebral scale system to measure canine heart size in radiographs. Journal of the American Veterinary Medical Association. 1995; 206:194-199.

2. Pinto ACBCF. Radiologia. In: Cubas ZS, Silva JCR, Catão-Dias JL. Tratado de animais selvagens. São Paulo: Roca; 2007: p 896-919.

3. Reichle JK, Wisner ER. Non-cardiac thoracic ultrasound in 75 feline and canine patients. Vet. Radiol. Ultrasound. 2000; 41:154-162. 
4. Gabay A. Radiologia Cardiovascular. In: Belerenian GC.Afecções Cardiovasculares em Pequenos animais. Ed. São Caetano do sul: Interbook; 2003: p 40-45.

5. Gülanber EG, Gönenci R, Kaya Ü, Aksoy Ö, Biricik HS. Vertebral scale system to measure heart size in thoracic radiographs of Turkish Shepherd (Kangal) dogs. Turkish Journal of Veterinary and Animal Sciences. 2005;29;723-726.

6. Lawler JV, Endy TP, Hensley LE, Garrison A, Fritz EA, Lesar M, Baric RS, Kulesh DA, Norwood DA, Wasieloski L.P., Ulrich M.P., Slezak T.R., Vitalis E., Huggins J.W., Jahrling P.B. \& Paragas J. Cynomolgus macaque as an animal model for severe acute respiratory syndrome. Plos Medicine.2006. 3;677-686.

7. Schillaci MA, Parish S, Jones-Engel L. Radiographic measurement of the cardiothoracic ratio in pet macaques from Sulawesi, Indonesia. Radiography. 2009; 15:29-33.

8. Rocha-Neto HJ, Moura LS, Pessoa GT, Ambrosio CE, Sousa FCA, Rodrigues RPS, Guerra PC, Alves FR. Cardiothoracic ratio and vertebral heart size (VHS) to standardize the heart size of the tufted capuchin (Cebus apella Linnaeus, 1758) in computerized radiographic images. Pesq Vet Bras. 2015; 35:853-858.

9. Wagner, WM, Kirberger, RM. Radiographic Anatomy of the Thorax and Abdomen of the Common Mormoset (Callithrix jacchus).Veterinary Radiology \& Ultrasound. 2005; 46:217-224.

10. Ji Y, Xie L, Liu S, Cheng K, Xu F, Li X, Wang T, Zhou Q, Fang L, Xie P. Correlation of thoracic radiograph measurements with age in adolescent Chinese rhesus macaques (Macaca mulatta). J. Am. Assoc. Lab. Anim. Sci. $2013 ; 52: 78-82$.

11. Xie L, Zhou Q, Liu S, Wu Q, Ji Y, Zhang, Xie, P. (2014). Normal thoracic radiographic appearance of the cynomolgus monkey (Macaca fascicularis). PloS one. 2014 9, e84599.

12. Alves FR, Costa FB, Machado PP, Diniz AN, Araujo AV, Ambrósio CE, Guerra PC. Anatomical and radiographic appearance of the capuchin monkey thoracic cavity (Cebus apella). Pesq Vet Bras. 2012; 32:1345-1350.

13. Felkai A, Vogelnest L, McNabb S, Allan G, Sangster C. Dilated cardiomyopathy in a De Brazza's monkey (Cercopithecus neglectus). J. Med. Primatol.2014; 43:209-212.

14. Dimopoulos K, Giannakoulas G, Bendavan I, Liodakis E, Petraco R, Diller GP, Piepoli MF, Swan L, Mullen M, Best N, Poole-Wilson PA, Francis DP, Rubens MB, Gatzoulis MA. Cardiothoracic ratio from posterior-anterior chest radiographs: a simple, reproducible and independent Market of disease severity and outcome in adults with congenital heart disease. Int. J. Cardiol. 2013; 166:453-457.

15. Charlier, MGS. Avaliação da função cardíaca de bugio ruivo (Alouatta guariba clamitans) em cativeiro. 2016.

16. Makungu M, Du Plessis WM, Barrows M, Groenewald HB, Koeppel KN. Radiographic thoracic anatomy of the ring-tailed lemur (Lemur catta). Journal of Medical Primatology 2014; 43: 144-152.

17. Young AN, du Plessis WM, Rodriguez D, Beierschmitt A. Thoracic radiographic anatomy in vervet monkeys (Chlorocebus sabaeus). Journal of Medical Primatology 2013; 42(6):310-7. 